This is the post print version of the article,

which has been published in IEEE journal of biomedical

and health informatics. 2018, 22 (4),1157-1167

http://dx.doi.org/10.1109/JBHI.2017.2757530.

\title{
Detection of snores using source separation on an Emfit signal
}

\author{
Jose Maria Perez-Macias* Student Member, IEEE, Mirja Tenhunen, Alpo Värri, Sari-Leena Himanen, \\ Jari Viik, Senior Member, IEEE
}

\begin{abstract}
Snoring (SN) is an early sign of upper airway dysfunction, and it is strongly associated with obstructive sleep apnea (OSA). SN detection is important to monitor SN objectively and to improve the diagnostic sensitivity of sleepdisordered breathing (SDB). In this study, an automatic snore detection method using an Emfit (Electromechanical film transducer) signal is presented. Representative polysomnographs of normal breathing (NB) and $\mathrm{SN}$ periods from 30 subjects were selected. Individual SN events were identified using source separation applying nonnegative matrix factorization deconvolution (NMFD). The algorithm was evaluated using manual annotation of the polysomnographic recordings. According to our results, the sensitivity (Se), and the positive predictive value (PPV) of the developed method to reveal snoring from the Emfit signal were $83 \%$ and $86 \%$, respectively. Compared to other approaches, our method adapts to the individual spectral snoring profile of the subject rather than matching a particular spectral profile, estimates the snoring intensity, and obtains the specific spectral profile of the snores in the epoch. Additionally, no training is necessary. This study suggests that it is possible to detect individual SN events with Emfit mattress, which can be used as a contactless alternative to more conventional methods such as piezo-snore sensors or microphones.
\end{abstract}

Index Terms - sleep, sleep-disordered breathing (SDB), snoring (SN), Emfit (Electromechanical Film Transducer) mattress sensor, nonnegative matrix factorization (NMF), nonnegative matrix factorization deconvolution (NMFD).

This research was supported by the Vilho, Yrjö ja Kalle Väisälän Foundation, and by Competitive State Research Financing of the Expert Responsibility area of Tampere University Hospital (grants 9P013, 9R007, 9S007).

Jose M. Perez-Macias, Jari Viik and Alpo Värri are with the Faculty of Biomedical Sciences and Engineering, University of Technology, Tampere, 33720 Finland, jose.perez-macias.eng@ieee.org.

Mirja Tenhunen is with the Department of Clinical Neurophysiology, Medical Imaging Centre and Hospital Pharmacy, and the Department of Medical Physics, Tampere University Hospital, Medical Imaging Centre, Pirkanmaa Hospital District, Tampere, Finland.

Sari-Leena Himanen is with the Faculty of Medicine and Life Sciences, University of Tampere, and the Department of Clinical Neurophysiology, Medical Imaging Centre and Hospital Pharmacy, Pirkanmaa Hospital District, Tampere, Finland.

\section{INTRODUCTION}

$\mathrm{S}_{\text {noring (SN) is one of the first signs of upper airway }}$ dysfunction and can be linked closely to sleep-disordered breathing (SDB) such as obstructive sleep apnea (OSA) [1][3]. Among adults, the prevalence of SN has been found to be $10-60 \%$, and it is more commonly found in men than women [4]-[6]. It is shown that snoring increases with age and the gender difference decreases as with aging [5], [7]. The vibrations and sound disturbances caused by SN have significant effects on the snorer's health and influence their bed partners quality of sleep too [8]. Several studies have associated SN with sleepiness, hypertension, angina pectoris, and cerebral stroke [4], [5]. However, Gottlieb et al. [2], suggested that these health problems could be explained by its close relationship with OSA. Also, simple SN has been associated with sleepiness, reduced work performance, and traffic accidents [6], [9]-[11]. Nevertheless, health consequences of SN without OSA are less clear [12]; Marin et al. [13] concluded there are no cardiovascular outcomes associated with SN [13]. However, other studies present contradicting results [14]. On the other hand, the mechanical vibration induced by SN may cause neurogenic lesions in the upper airway tissues [15], carotid atherosclerosis [16], inflammatory responses in a intracellular level [17], and vascular smooth muscle cell injuries [18]. Furthermore, the loud sound and vibrations might cause arousals to the snorer as well as to the bed partner [8], impeding an uninterrupted and restorative sleep [19].

The Gold standard for SDB diagnostics in the clinical practice is done using polysomnography (PSG). In the clinical practice, the American Association of Sleep Medicine (AASM) recommends three methods to detect SN [20]: a piezoelectric sensor placed on the neck, an acoustic sensor such as microphone on the trachea, chest or near the patient's bed, and nasal prongs connected to the nasal pressure sensor. Arnardottir et al. [21] advocated the use of audio-based techniques over others and encouraged the development of a standardized method to measure SN. However, these methods may have some drawbacks. They are annoying and may disturb patient's sleep. For instance, in the case of oral breathing or under the presence of moisture in the nasal prongs, the SN detection is ruined. Furthermore, the piezoelectric sensor may entirely or partially detach during the 
TABLE I

DEMOGRAPHIC AND CLINICAL DATA FROM THE SUBJECTS UNDER STUDY (24 MEN, 6 FEMALE)

\begin{tabular}{lllll}
\hline \hline Parameter & Units & $\begin{array}{l}\text { Min- } \\
\text { max }\end{array}$ & Median & IQR \\
\hline Age & {$[\mathrm{yrs}]$} & $25-60$ & 44.5 & 16.5 \\
Body mass index & {$\left[\mathrm{Kg} \cdot \mathrm{m}^{-2}\right]$} & $22.2-54$ & 29 & 8.4 \\
Snore time & {$[\mathrm{min}]$} & $0-286$ & 30 & 84 \\
Apnea-hypoapnea index & - & $0-105.7$ & 15 & 29.1 \\
\hline
\end{tabular}

night due to movement or sweat artifacts, leaving this sensor impractical for SN detection. Also, even when employing external microphones offer a high-quality measure of the characteristics of SN, its setting is complex and dependent on the distance from the patient.

The Electromechanical Film Transducer (Emfit) is a permanently charged polypropylene ferro-electret film sensitive to mechanical stress. The polymer layers are separated by air gaps that upon an orthogonal mechanical force, the charge differential between these gaps and the permanently charged internal layers, produces a change of potential. As a result, the Emfit signals is a consequence of pressure changes inducing potential differences, which are measured with a differential AC amplifier [22], [23]. The high sensitivity of the Emfit sensor allows place it unobtrusively under the mattress.

Unobtrusive sensors, such as Emfit, promise to ease and complement PSG diagnostics. Mattress type sensors like Emfit, the Polyvinylidene fluoride film (PVDF), the staticcharge-sensitive bed (SCSB), the sheet-type SD-101, and the balancing tube air-mattress (AMBT), have been used for multiple purposes, such as (1) breathing rate (BR) and heart rate (HR) monitoring [24]-[29], (2) SDB diagnosis [30]-[40], (3) epilepsy monitoring, (4) periodic leg movements and restless legs syndrome assessment [41]-[43]. In addition to solve the aforementioned issues in the normal clinical settings, mattress-like sensors provide additional physiological information; namely, HR and breathing. Moreover, Emfit reveals the increased effort present in periods with prolonged partial obstruction [30].

Despite the advantages of mattress-like sensors, few studies exist about SN detection using them. Hwang et al. [44] proposed an automatic SN detection algorithm using a PVDFtype mattress sensor based on two spectral features of SN events (power ratio and peak frequency) and machine learning techniques. Also, Shin et al. presented simple filtering and thresholding techniques to detect SN on an AMBT [40]. These methods rely on selected spectral features and need training to function. Compared to other mattresses, the Emfit mattress is more sensitive than PVDF mattress in the orthogonal direction, and less than $1 \%$ of the signal is affected by lateral forces [23].

In our previous study, we characterized the spectral differences of thirty-seconds epochs length from NB and SN periods in the Emfit signal using the power spectrum [32]. We wanted to continue to develop unsupervised methods to detect individual snores based on source separation techniques using spectrogram factorization. Mono-source separation techniques have been traditionally employed in music [45], [46] and more
TABLE II

SUMMARY OF ANNOTATED EVENTS PER PATIENT IN THE STUDY

\begin{tabular}{llclll}
\hline \hline & $\begin{array}{l}\text { Total } \\
\text { amount }\end{array}$ & Mean \pm SD & Min-max & Median & IQR \\
\hline SN events & 3521 & $117.4 \pm 54.4$ & $36-245$ & 115 & 96 \\
Artifacts & 16 & - & $0-2$ & 0 & 1 \\
\hline
\end{tabular}

SN events, snoring events; SD, standard deviation; min, minimum; max, maximum; IQR, interquartile range.

recently, to detect $\mathrm{HR}$ and breathing using a digital stethoscope [47]. The objective of this study is to investigate the use of Emfit mattress for snoring assessment by using advanced signal processing methods. Additionally, we want to find out the spectral profiles of snoring in the Emfit sensor signal using source separation techniques.

\section{DATA ACQUISITION AND STUDY DESIGN}

\section{A. Subjects}

Thirty-three patients under suspicion of SDB took part in the study, including one-night full PSG. Three recordings had to be omitted from the study due to high-level noise, or electrical artifacts in the Emfit signal. The final data set consisted of 30 PSGs. The age of the subjects ( 24 men, 6 women) ranged from 25 to 60 years. Anthropometric data are shown in Table 1. All patients had signed a written informed consent before recordings were performed in the sleep laboratory of Tampere University Hospital, Tampere, Finland. This study was approved by the Ethical Committee of the Pirkanmaa Hospital District.

\section{B. Recording}

PSGs were performed using a standard monitoring montage

(a)

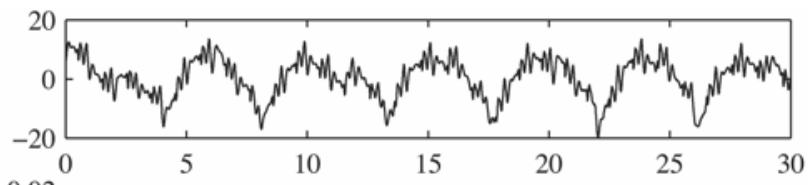

(b)

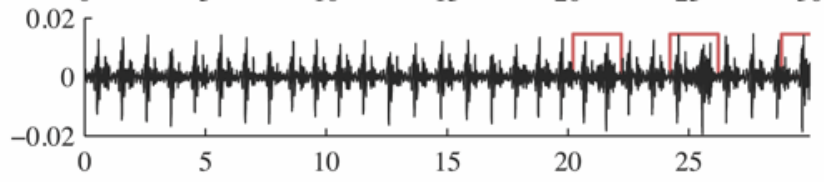

(c)

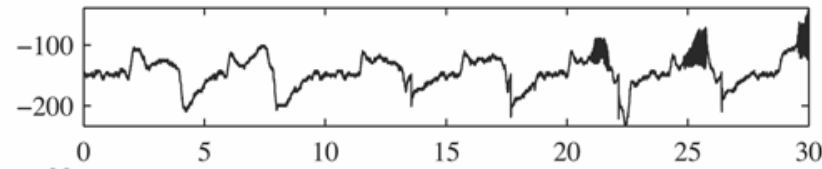

(d)

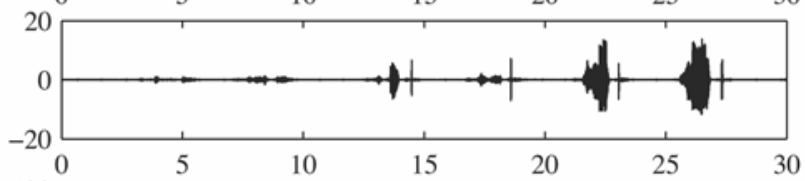

(e)

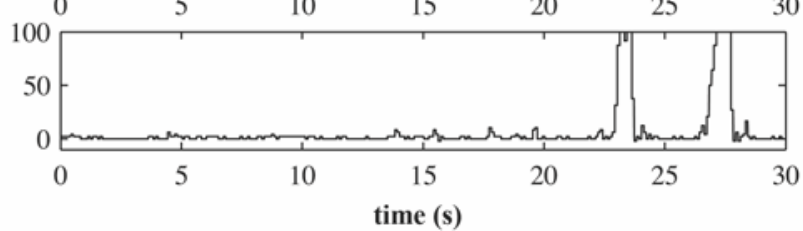

Fig. 1: Thirty-seconds epoch with three snoring (SN) events for the (a) Emfit (mV); (b) band-pass filtered Emfit (6-100 Hz, mV); (c) nasal pressure ( $\mu$ bar); (d) tracheal sound signals (V); and (e) envelope trace of piezoelectric sensor (units). Scored SN events are represented as red rectangles. 
including EEG (F3-A2, F4-A1, C3-A2, C4-A1, O1-A2, O2A1); electromyographs of submental and tibialis muscles, and the two channels of the electrooculogram; an electrocardiogram; pulse oxygen saturation $\left(\mathrm{SpO}_{2}\right)$; and body position measurements. A nasal air pressure transducer was employed to measure the airflow. Respiratory movements were assessed with thoracic and abdominal inductive belts. SN was measured with a piezoelectric sensor situated on the neck and a tracheal microphone positioned above the suprasternal notch. The Emfit mattress sensor of dimensions $32 \mathrm{~cm} \times 62$ $\mathrm{cm} \times 0.4 \mathrm{~cm}$ was placed under the thoracic area of the sleeping patient under the mattress. Data were recorded with an Embla N7000 and Somnologica Studio 3 software setup (Embla ${ }^{\circledR}$, USA). The sampling rate of $2 \mathrm{~Hz}$ was used for $\mathrm{SpO}_{2}, 10 \mathrm{~Hz}$ for respiratory movements, $500 \mathrm{~Hz}$ for the electrocardiogram (ECG), $100 \mathrm{~Hz}$ for the piezoelectric snore sensor, $11 \mathrm{kHz}$ for the tracheal microphone, and $200 \mathrm{~Hz}$ for the Emfit sensors, and all other signals.

\section{Data selection and snore episode reference labeling}

Representative periods of SN and NB of a maximum duration of $10 \mathrm{~min}$ per patient were selected by an experienced neurophysiologist. The scoring of the SN periods was based on three different channels. The airflow channel inferred from the nasal air pressure signal was used to preselect SN periods based on the high-frequency SN components on top of the airflow waveform. During these preselected periods SN had to appear in the piezo-sensor signal placed on the neck (envelope technique, threshold $10 \mathrm{uV}$ ). Following, SN was confirmed by the signal of the tracheal microphone (Fig.1). Finally, SN sounds were listened from the video file. NB periods did not contain SN, apneas or hypopneas in any of the previously above signal characteristics. In addition, each patient SN intensity was evaluated and annotated by an independent expert scorer as low $(\mathrm{L})$, medium $(\mathrm{M})$, and high $(\mathrm{H})$ on the 10 min SN periods based on the video file. To ease the processing, NB and SN periods were divided into thirtyseconds epochs. The total number of epochs were 1119, of which 473 and 546 were NB and SN epochs, respectively. Visual inspection of each epoch was performed to annotate the artefacts in the Emfit signal; detected events concurring with the annotated artefacts were discarded. The percentage of epochs of male subjects for NB epochs and SN epochs were 78.6, and 79.3\%, respectively.

In a second scoring step, snore events were annotated by an independent expert scorer from each selected period. The same protocol used in the period selection step was followed. Additionally, if SN was present during either inspiratory and or expiratory phase of the breathing cycle, the event was annotated as a single SN event. The total annotated SN events were 3521 (mean \pm standard deviation; $117.4 \pm 54$.4). Table 2 reports the summary of the scored snore events. Fig. 1 depicts a thirty-seconds example of an epoch with two SN events.

\section{METHODS}

The architecture of the proposed algorithm is illustrated in Fig. 2. It comprises of four stages: preprocessing (Fig. 2-1), quality assessment (Fig. 2-2), SN detection (Fig. 2-3), and evaluation (Fig. 2-4).

\section{A. Preprocessing}

The Emfit signal $(r[n])$ was high-pass filtered using a finite impulse response (FIR) filter designed with a Hamming window, cut-off frequency at $6 \mathrm{~Hz}$, and $1 \mathrm{~Hz}$ transition band. This filter removes the high power heart components under 6 $\mathrm{Hz}$, the baseline, and the breathing signal.

Acquired data in clinical environments are often noisy. We modeled our signal $u[n]$, as a sum of the desired signal $d[n]$ and noise $\eta[n]$ : additive white Gaussian noise (AWGN) $\left(\eta_{A W G N}\right)$ and power line interference $\left(\eta_{P L I}\right)$, and other noise

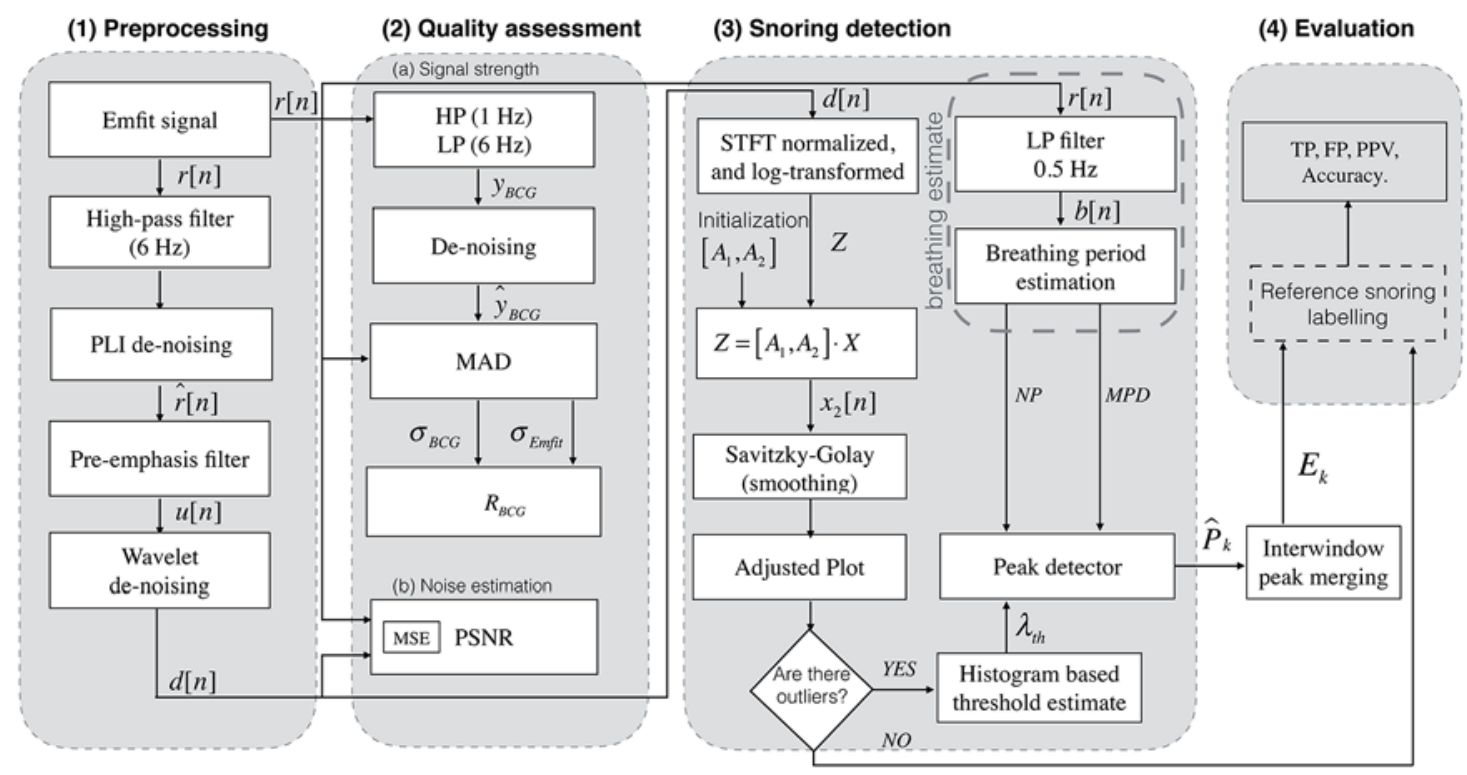

Fig. 2: Block diagram of the snoring detection process: (1) pre-processing and signal conditioning, (2) quality assessment, (3) snoring detection, and (4) evaluation. HP, high pass; LP, low pass; MAD, mean absolute deviation; PSNR, peak signal to noise ratio; STFT, short-time Fourier transform; TP, true positives; FP, false positives; PPV, positive predictive value; NP, number of peaks; MPD, minimum peak distance. 
$\left(\eta_{o}[n]\right)$.

$$
r[n]=d[n]+\eta_{A W G N}[n]+\eta_{P L I}[n]+\eta_{o}[n]
$$

Before the acquisition, the signal is subject to frequencydependent attenuation when transmitted through the body, bed sheets, sleeping mattress, and the losses in the material transitions. To partially compensate the attenuation and enhance the frequencies where SN occurs, the signal was filtered with a pre-emphasis filter. This filter emphasizes highfrequency components while attenuating lower frequency components. The employed filter is a first order FIR filter defined in (2), where the constant $a$ was set to 0.97. The Emfit signal was filtered with a pre-emphasis filter after PLI noise removal.

$$
u[n]=\hat{r}[n]-a \cdot \hat{r}[n-1],
$$

where $\hat{r}[n]$ represents the band-passed acquired data.

The PLI was removed using an adaptive algorithm centered at $50 \mathrm{~Hz}$ [48]. To remove the Gaussian noise, we used a wavelet denoising technique using a 15-level 13th order Symmlets using soft-thresholding, Heursure method for threshold selection, with unscaled noise modeling and threshold rescaling using the first level coefficients. This approach has been used to denoise phonocardiogram [49].

\section{B. Signal quality estimation: signal strength and noise}

To study the dependence of our algorithm with the signal strength and noise we estimated two measures. To measure the strength of the signal, the ratio between a denoised ballistocardiogram (BCG) and the raw Emfit signal (3) was estimated from each epoch for each patient. The noise was quantitatively evaluated using the peak signal-to-noise ratio (PSNR) (4). The BCG signal $\left(y_{B C G}\right)$, was extracted using a high-pass Butterworth filter with cut-off frequency of $1 \mathrm{~Hz}$ to remove the low-frequency respiratory components [25], and a low-pass filter with cut-off frequency of $5 \mathrm{~Hz}$.

The denoised BCG signal was estimated using a Wavelet denoising technique using a 5-level fourth order Symmlets, using soft-thresholding, Heursure method for threshold selection; this denoising technique has been used successfully in ECG denoising [50].

$$
\begin{gathered}
R_{B C G}(d B)=10 \cdot \log \frac{\sigma_{B C G}^{2}}{\sigma_{\text {emfit }}^{2}}, \\
P S N R=10 \cdot \log \frac{r_{\text {max }}}{M S E} \\
M S E=\frac{1}{N} \sum(r[n]-d[n])^{2},
\end{gathered}
$$

where $\sigma^{2}$ is the variance estimated using the mean absolute deviation (MAD) as a robust estimator of the variance [51], [52], MSE is the mean squared error, and $u_{\max }$ is the maximum value of $u[n]$.

\section{Source separation using non-negative matrix factorization deconvolution (NMFD)}

The goal of applying non-negative factorization (NMF) in an Emfit spectrogram is to bring out multiple sources (or signals of interest e.g. SN) from a single-channel mixture of signals. We considered the Emfit signal as an additive combination of several components: the pulse wave caused by the beating heart or BCG, SN, and noise.

$$
r[n]=\sum_{i} s_{i}[n]+\eta[n],
$$

where $i$ corresponds to the $i$-th source, $s_{i}[n]$ the source signal, and $\eta[n]$ represents the AWGN and PLI noise, and other unwanted noise.

The Emfit signal can then be represented using a timefrequency representation using the spectrogram or short-time Fourier transform (STFT) (7). The spectrogram provides a representation of the signal power distribution over a set of frequencies at specific time windows.

$$
Y(n, \omega)=\sum_{m=-\infty}^{\infty} d[m] w_{\text {Hamm }}[n-m] e^{-j \omega m},
$$

where $w_{\text {Hamm }}$ is the Hamming window. The magnitude spectrogram $(Z=|Y(n, \omega)|)$ is estimated calculating the absolute value of the STFT. From now, we refer to $Z$ as a matrix with $m$ rows and $n$ columns. The magnitude spectrogram was normalized by the median energy contained within the $6-10 \mathrm{~Hz}$ band of the spectrogram (8), using this band as reference intensity for all patients because it contains heart-originated activity present in all subjects. Finally, the Z is log-transformed:

$$
Z_{n}=\log \left(Z(m, n) / \sum_{m \sim 6 H z}^{m \sim 10 H z} Z(m, n)\right)
$$

The source separation is accomplished factorizing the magnitude spectrogram in two matrices (9), in such a way that the result groups the components into the sources.

$$
Z=A^{\prime} \cdot X^{\prime}
$$

One of the applications of matrix factorization is blind source separation (BSS) where the observations are considered as a weighted sum of $n$ underlying sources. NMF has been used in single-channel source separation of audio source [53], drum transcriptions [46], and recovery of biological signals [47]. Inspired by previous work by Paatero et al. [54], NMF was first proposed as an algorithm to learn parts of faces and text semantics [55], [56]. The factorization assumes a compositional model of audio sources, i.e. an additive combination of components that do not result in subtraction or diminishment of any of the parts [57].

NMF is applied to the magnitude spectrogram of the signal $Z \in \mathcal{R}_{+}{ }^{F \times T}$, where $\mathcal{R}_{+}$represents the set of non-negative real numbers, $T$ the number of time frames, and $F$ is the resolution of the discrete Fourier transform (DFT). The goal of NMF is 
to approximate $\mathrm{Z}$ as a product of two non-negative matrices (9), by minimizing the error of reconstruction.

$$
Z \approx A \cdot X
$$

Each spectral vector $y_{t}$ is thus a linear combination of all the rows or atoms $a_{s}$ (in the $s$-th atom in time $n$ ), and the time varying activation $x_{s}[n]: y_{t}=\sum_{s=1}^{S} a_{s} x_{s}[n], s=1 \ldots S$. The number of atoms $S$ is usually chosen so that $(F \times T)<F T$, so the product can be regarded as a compressed form of $Y$. There are several proposed NMF algorithms. The classic NMF factorization algorithm minimizes the Kullback-Leibler (KL) divergence [55]:

$$
\mathrm{A}^{*}, X^{*}=\underset{A, X}{\operatorname{argmax}} D(Z \| A X), A \geqslant 0, X \geqslant 0,
$$

where $\mathrm{A}^{*}$ and $X^{*}$ are the optimal values of $\mathrm{A}$ and $\mathrm{X}$ from the minimization problem. However, this factorization does not take into account the relative positions of each of the atoms and consequently ignores temporal information. An extended version of the NMF called NMFD initially proposed by Smaragdis (2004) takes into account temporal information in the factorization process [58]. The expression $\mathrm{xx}$ is then transformed into:

$$
Z \approx A \odot X
$$

where $\odot$ is the circular convolution. The target is to minimize a new cost function:

$$
\mathrm{D}=\left\|Z \otimes \frac{\ln \mathrm{Z}}{\Lambda}-Z+\Lambda\right\|_{F}^{\prime}
$$

with $\Lambda=A \odot X,\|\cdot\|_{F}$ is the Frobenius norm, and $\otimes$ is the Hadamard product (an element-wise multiplication). A detailed description and the multiplicative updates for estimating $A$, and $X$ is described by Smaragdis et al. [58].

The NMFD is applied to the Emfit signal and restricted to decompose in two signals (number of atoms or $S$ ). Traditionally, the matrix decomposition is initialized creating a random $A$ matrix $\left(A_{s}^{F \times C_{i}}=A^{F \times\left(C_{1}+C_{2}\right)}\right)$, where $C_{i}$ represents columns $i=1 \ldots S$. In this study, we initialized each atom of matrix $A$ to those frequencies that matches our source signal. Previous work [32], found a significant power increase in the bands [16-40] and [55-100] $\mathrm{Hz}$, thus we initialized the $C_{2}$ components to be maximum at frequencies at $30-100 \mathrm{~Hz}$, and $C_{1}$ components to be maximum at $6-30 \mathrm{~Hz}$ :

$A_{s}^{F \times C_{i}}=a_{i j}=\left\{\begin{array}{c}a_{i 1}=\alpha_{1} \forall i, f_{i} \in[6-30] \mathrm{Hz}, 0 \text { otherwise } \\ a_{i 2}=\alpha_{2} \forall i, f_{i} \in[30-100] \mathrm{Hz}, 0 \text { otherwise }\end{array}\right.$

where $\alpha_{l}$ for $l=[1,2]$, and $F$ being the frequency resolution of the STFT. These step functions were convolved with a Gaussian kernel to smooth the transition between zero values and alpha values. The NMFD algorithm iteratively adjusts the columns $\left(a_{1-2}=C_{1-2}\right)$ to minimize the cost function. The NMFD implementation has the added constraint consisting on maintaining the initial zero values of matrix $A$ during the multiplicative updates.

The snoring events $(E)$ are the result of finding the peaks $\hat{P}_{k}$ in the time varying activation vector $x_{2}[n]$, where $s=2$ corresponds to the atom associated with snoring $\left(y_{t}=\right.$ $\left.a_{2} x_{2}[n]\right)$.

\section{Snoring event detection}

The activation matrix component corresponding to the snoring source is then $x_{2}[n]$. To remove spurious peaks from $x_{2}[n]$, a Savitzky-Golay fourth-order FIR filter [59] of one second length was applied. The one second time-length, corresponds to the "typical" minimum time of a SN event. This smoothing technique is based on least-squares fitting of polynomials to the data segments, preserving the shape of the activation vector $x_{2}[n]$.

Under the presence of snores, the activation vector $x_{2}[n]$ has two different distributions. It was observed that on the absence of snores, the spectral profile $a_{2}[n]$, is small, and approximately flat. The activation vector exhibited an approximately Gamma distribution. Thus, we made the assumption that the activation vector $x_{2}[n]$, follows a slightly Gamma distribution. Conversely, under the presence of snoring events, the distribution becomes more skewed, following a generalized extreme value distribution (GEV) or when the snores loud and long, it becomes a Gaussian mixture of two components. We assumed these models to assess the presence of events within the epoch. The model selection was based on the second-order Akaike Information Criterion (AIC) [60], [61]. If the criterion resolves on a distribution associated with events, the algorithm continues to the next step. Otherwise, the algorithm considered the epoch as NB and proceeds to proceeds to the next epoch. 

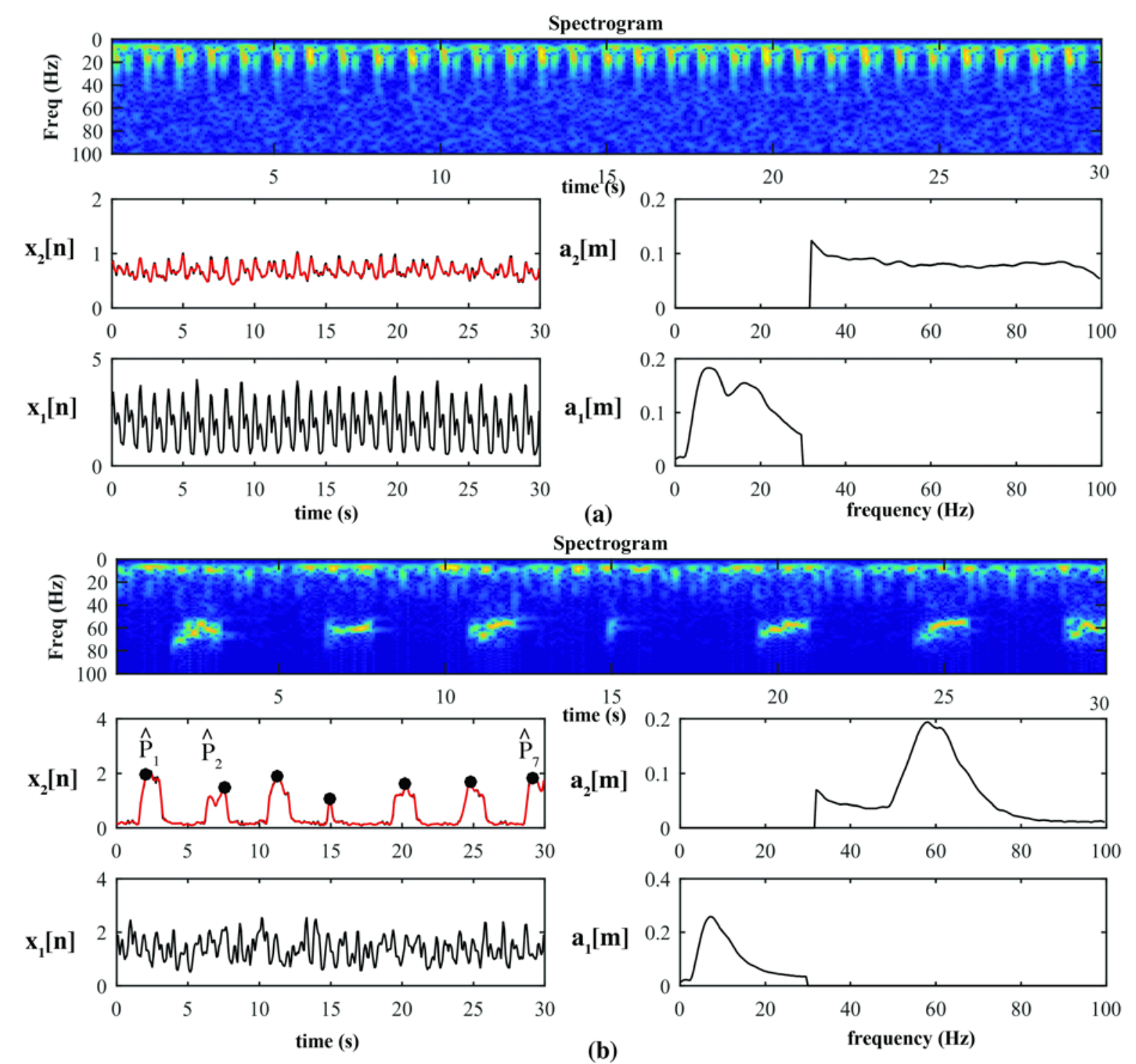

Spectrogram

Fig. 3: Spectrogram factorization normal breathing epoch (NB), and (b) of a snoring epoch (SN). Activation matrices $\left(x_{s}[n], s=[1,2]\right)$ of the first and second components, respectively. Atoms/spectral profiles $\left(a_{s}[n], s=[1,2]\right)$ of the activation matrices. In black, $x_{2}[n]$; in red, the output of the Savitzky-Golay FIR filter. The black dots represent the peaks within the epoch $\widehat{P}_{k}$.

Once the presence of events in the activation vector is confirmed, the algorithm proceeds to the snore event detection. To detect a snore event $\left(E_{k}\right)$, a threshold $\left(\lambda_{t h}\right)$ on the activation vector must be estimated, manually or automatically. One of the main issues in event classification is to find a suitable threshold. Machine learning and statistical approaches derive a threshold that is dependent on the training data. These are inherently variant across subjects, although data is normalized to compensate. In this work, we used an adaptive threshold based on the current epoch. Histogram based methods are dependent on the distribution of the activation vector on each epoch. These algorithms have been heavily studied for image segmentation and binarization. We implemented a modified thresholding technique based on the shape of the histogram's derivative [62]. However, instead of smoothing the signal's histogram of each frame, as is done by Taskanen et al. (2015), we fitted a kernel distribution to the activation's matrix histogram $\left(x_{2}[n]\right)$. Subsequently, we estimated the first derivative to estimate the global minimum, and then we estimated the next local peak towards infinity. This approach yields more robust results and increases sensitivity considerably. Also, if the next local peak is not found, the epoch is discarded for further processing.

Finally, peaks were detected using the built-in MATLAB ${ }^{\circledR}$ function findpeaks.m. This built-in algorithm has three inputs, the number of peaks (NP), the minmun peak distance (MPD), and maximun peak height (MPH). The input parameters NP and MPD we estimated from the breathing rate derived from the raw Emfit signal. To estimate the breathing rate, we extracted the breathing waveform from the raw Emfit signal by filtering $\mathrm{u}[\mathrm{n}]$ with a 3rd-orther Butterworth low pass filter with a cut-off frequency of $0.5 \mathrm{~Hz}$. Each epoch of the resulting breathing waveform $(b[n])$ was interpolated using weighted linear least squares and a 2nd degree polynomial model over $(b[n])$. This interpolation method has the advantage to fit the 

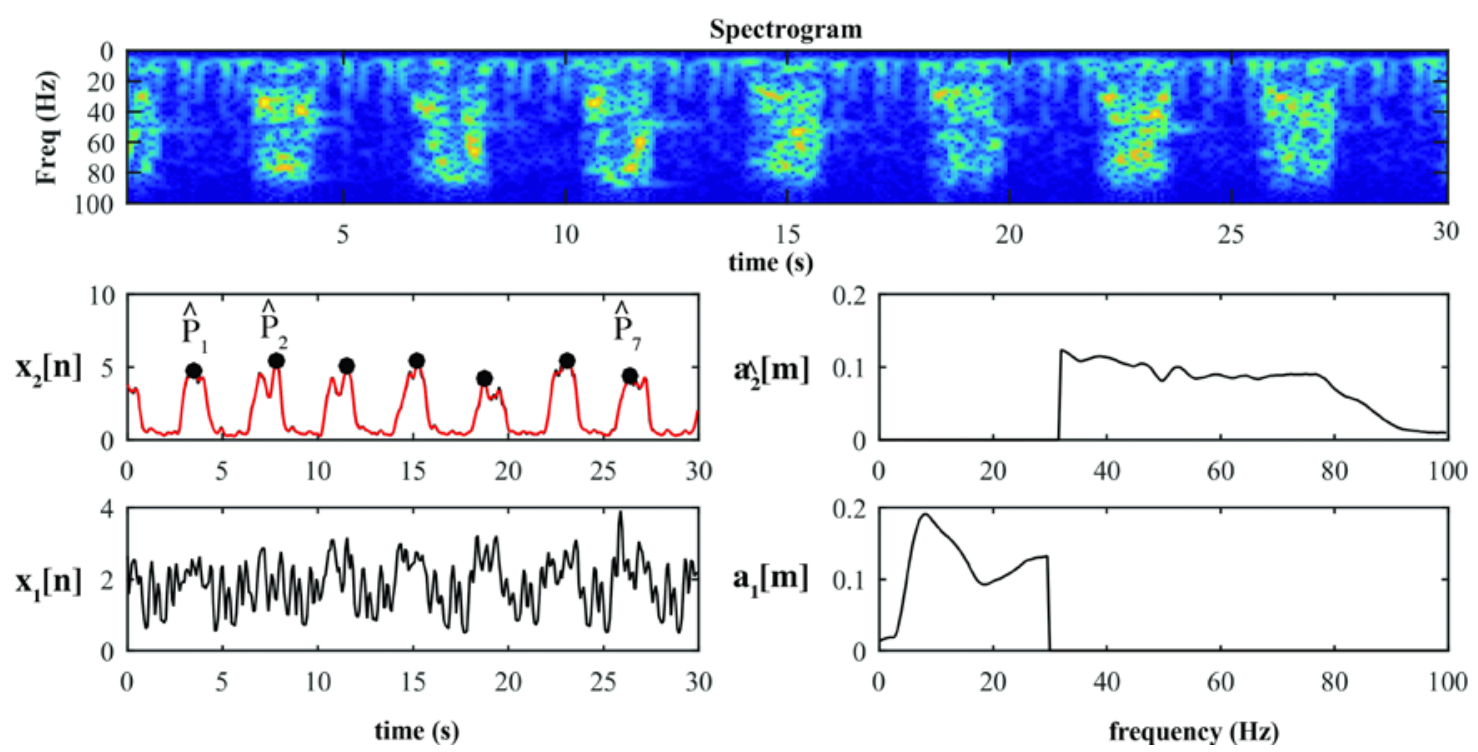

Fig. 5: Spectrogram factorization of a snoring epoch with another snoring profile. Activation matrices $\left(x_{s}[n], s=[1,2]\right)$ of the first (HR), second (SN) components, respectively. Atoms/spectral profiles $\left(a_{s}[n], s=[1,2]\right)$ of the activation matrices. In black, $x_{2}[n]$; in red, the output of the Savitzky-Golay FIR filter. The black dots represent the peaks within the epoch $\hat{P}_{k}$.

breath to a quasi-sinusoidal pattern while preserving breathing cycles. The number of peaks and valleys to detect were calculated by estimating breathing period with autocorrelation analysis. A 20 percent error margin for both NP and MPD was assumed to account for the intra-epoch breathing variability. The maximum peak height (MPH) corresponds to the estimated threshold, $\lambda_{t h}$. For each epoch we obtain a collection of peaks: $\hat{P}_{k}=\left[\hat{L}_{k}, \hat{I}_{k}\right]$ at locations $\hat{L}_{k}$, and their associated intensity value $\hat{I}_{k}, k=1 \ldots, K$, where $\mathrm{K}$ is the number of detected peaks.

The NMFD algorithm is applied to a fixed-length window of thirty-seconds with an overlap of $5 \mathrm{~s}$, and hence, several unwanted peaks are detected. We ignored the peaks when only one peak was found in the epoch. In our implementation, we merged those peaks resulting from the same snoring event resulting in $P_{k}=\left[L_{k}, I_{k}\right]$. Finally, the raw peak locations were refined such that each snoring event corresponded to the location taking the beginning of the recording as reference. The new re-referenced peaks $E_{k}=\left[L_{k}^{\prime}, I_{k}\right]$, where $\mathrm{E}$ symbolizes $k$-event at location $L_{k}^{\prime}$ with instensity $I_{k}$, are later evaluated with the annotated snore events.

\section{E. Data and Statistical Analyses}

Signal processing and statistical analyses were performed using MATLAB (R-2013b, The MathWorks, Inc., Natick, MA, USA). The NMFD algorithm was modified from an NMFLib by Graham Grindlay [63]. The performance of the algorithm was evaluated using the total number of true positives (TP, number of detected snores that correspond to annotated snores), the total false positives (FP, the number of detected snoring events that were not annotated), total false negatives (FN, number of annotated events that were not detected by the algorithm), the positive predictive value (PPV, proportion of SN epochs which are true positives) (15), the sensitivity (Sen, the TP rate of the algorithm as the ratio of TP to predict positives) (16), and the F-score (17). The resulting
F-scores are measures of the overall concordance between the results of the algorithm and the visual scores.

$$
\begin{gathered}
P P V=\frac{T P}{T P+F P} \\
\text { Sen }=\frac{T P}{T P+F N} \\
F=2 \cdot \frac{P P V \cdot S e n}{P P V+S e n}
\end{gathered}
$$

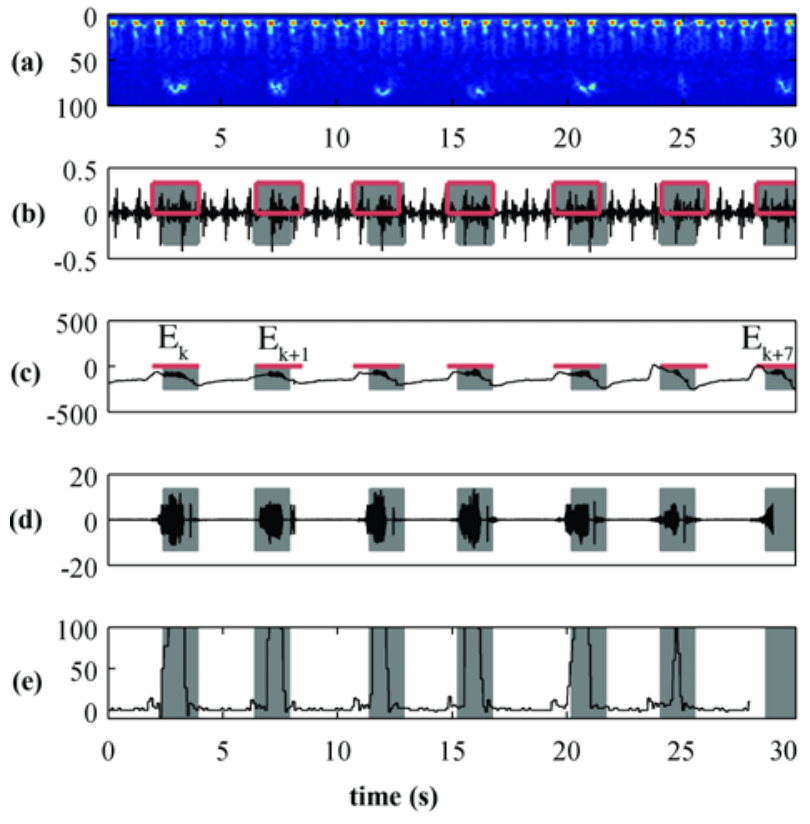

Fig. 4: Detected SN events (a) Emfit spectrogram; (b) band-pass filtered Emfit (6-100 Hz, mV); (c) nasal pressure ( $\mu$ bar); (d) tracheal sound signals (V); and (e) envelope trace of piezoelectric sensor (units). Scored $\mathrm{SN}$ events are represented as red rectangles and detected SN events $\left(E_{k}\right)$ in gray rectangles. 


\section{Results}

A Hamming window of length 64 samples and $75 \%$ overlap and a 512-point DFT was employed to estimate the spectrogram. To reduce the amount of computing time, the NMFD algorithm was allowed to run for maximally 500 iterations in all factorizations or until convergence as defined by the relative change in the cost function; also our tests found reasonable convergence at this point. The wavelet denoising technique was applied to epochs with signal strength lower than $-9 \mathrm{~dB}$, this level was estimated experimentally.

\section{A. Source separation of the Emfit signal}

The STFT was computed, normalized, and log-transformed; samples can be seen in Fig. 3 and 4 in the top rows. The resulting spectrogram was factorized using the NMFD, setting the number of components to $S=2$. One corresponding to $\mathrm{SN}$ and the other component for pulse wave signal from the heart. A representative NB epoch (Fig. 3a) and two SN epochs (Figs. $3 \mathrm{~b}$ and Fig. 4) were selected to visualize the result of NMFD. Subplots on the left side represent the time activations $x_{s}[n]$, $s=[1,2]$, and subplots on the right side represent the atoms $\left(a_{s}[n], s=[1,2]\right)$. Due to our initialization procedure of the NMFD algorithm, snoring always corresponds to the second component, $x_{2}[n]$. This spectral profile is in line with our previous study [32] where a similar power spectrum density (PSD) was found when comparing snoring and normal breathing PSDs. It is also consistent why power ratios perform well in certain SN profiles [44]. However, not all snores had the same spectral profile; Fig. 3b and Fig. 4 show the capability of the algorithm to adapt to the different spectral snore profiles (see components $a_{2}[n]$ in Fig. 3 and 4).

\section{B. SN event detection}

Fig. 3-4 display the activations for the SN component are shown $\left(x_{2}[n]\right)$. After the threshold $\left(\lambda_{t h}\right)$ was estimated for each epoch, the detected peaks $\left(\hat{P}_{k}\right)$, were merged from the different overlapping windows $\left(P_{k}\right)$. The merged peaks are considered the center of the snore events $\left(E_{k}\right)$ with a time duration of one second. A sample of detected peaks $\left(\hat{P}_{k}\right)$ is shown in Fig. 3b, and Fig 4.

\section{Evaluation results}

Fig. 5 shows the annotated events together with the detected events. Table 3 summarizes the individual results for the proposed method. SN intensities were summarized using minimum, maximum, median, and interquartile range. Median $\mathrm{R}_{\mathrm{BCG}}$ and PSNR values from SN epochs, apnea-hypoapnea index (AHI), and body mass index (BMI) are shown in the table. Lowest performances corresponded with lowest signal intensity and noise $\left(\mathrm{R}_{\mathrm{BCG}}\right)$. Especially noticeable was patient with Id. 18. A closer look at this signal showed high noise intensity and a weak signal. Thus, snores did not stand out.

TABLE III

CLASSIFICATION RESULTS OF SNORING EVENT DETECTION FROM EMFIT MATTRESS USING SOURCE SEPARATION TECHNIQUES

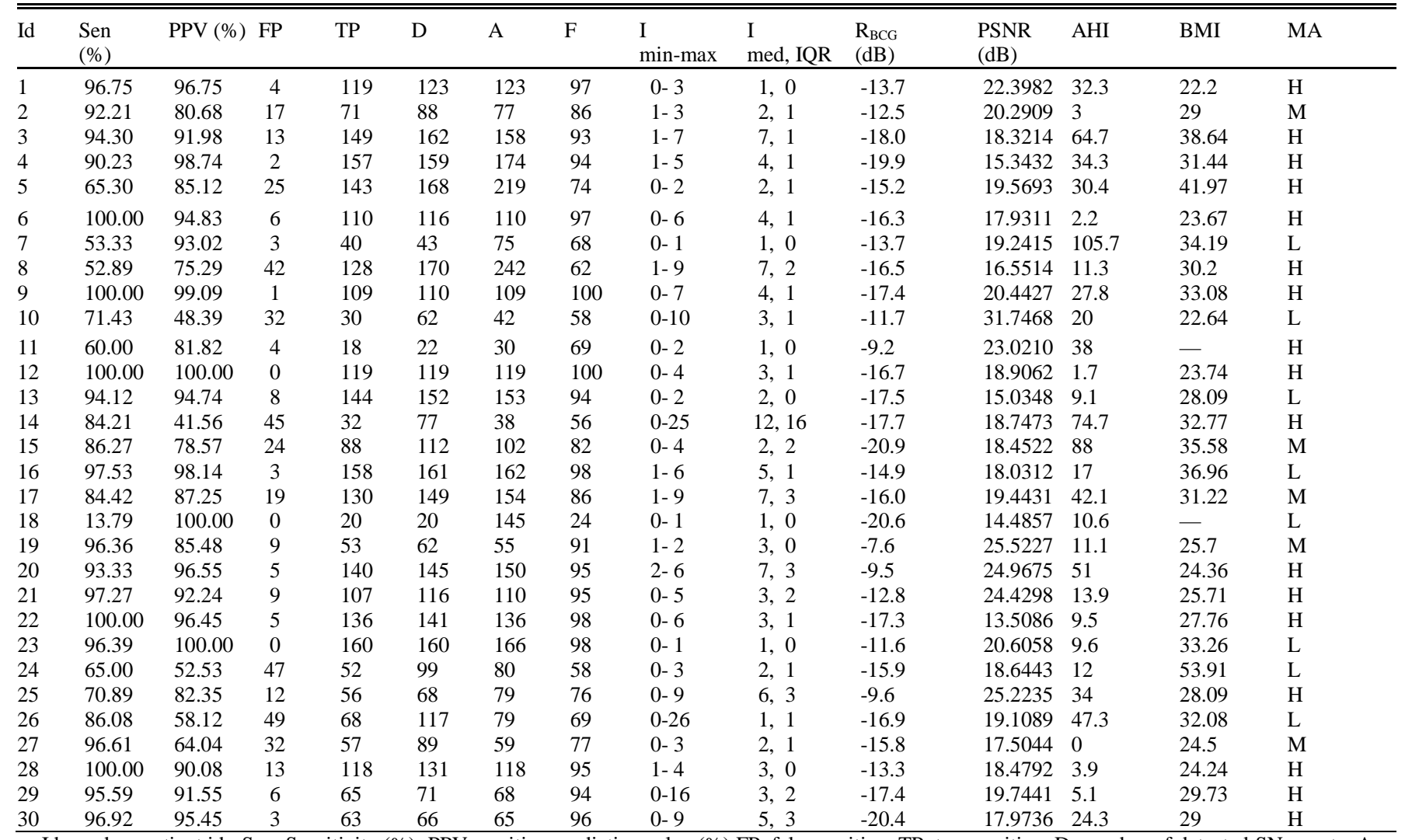

Id, random patient id.; Sen, Sensitivity (\%); PPV, positive predictive value (\%);FP, false positive; TP, true positive; D, number of detected SN-events; A, number of annotated SN-events; F, F-measure or balanced F-score; Imin, minimum intensity; Imax, maximum intensity; Imed, median intensity; IIQR, interquartile range intensity; $\mathrm{R}_{\mathrm{BCG}}$, Ratio between BCG and raw Emfit (dB); PSNR, peak signal to noise ratio; AHI, apnea-hypoapnea index, BMI, body mass index; MA, manual annotations for intensity of snoring. 
TABLE IV

CLASSIFICATION RESULTS FOR ALL SNORERS USING ( $1^{\mathrm{ST}}$ ROW), AND THE HIGH, MEDIUM AND LOW INTENSITY SNORERS.

\begin{tabular}{llllllll}
\hline \hline $\begin{array}{l}\text { Scored } \\
\text { intensity }\end{array}$ & Sen (\%) & PPV (\%) & FP & TP & D & A & F \\
\hline All & 82.81 & 86.29 & 447 & 2813 & 3260 & 3397 & 85 \\
\hline High & 86.79 & 91.94 & 163 & 1859 & 2022 & 2142 & 89 \\
\hline Medium & 90.00 & 81.57 & 122 & 540 & 662 & 600 & 86 \\
\hline Low & 63.21 & 71.88 & 162 & 414 & 576 & 655 & 67 \\
\hline
\end{tabular}

Sen, Sensitivity (\%); PPV=positive predictive value (\%); TP, true positive; FP, false positive; D, detected snoring (SN) events; A, annotated SN events; F, F-measure or balanced F-score.

Table 4 lists the results for all patients and the classification of high, medium and low intensity snorers given by the expert. The overall performance of the algorithm achieved Sensitivity and PPV of 82.81 and $86.29 \%$, respectively. Best detection performance was achieved in those patients where the intensity of SN was highest.

\section{DISCUSSION}

In the present study, we proposed a novel method to detect SN events, obtain their spectral profile and derived an SN intensity value using source separation techniques. The algorithm detects snores without training based on the detection of the spectral energy distribution of SN in the frequency band $30-100 \mathrm{~Hz}$.

Thirty-second long epochs with a $5 \mathrm{~s}$ window overlap were used in the study. The performance difference using other epoch sizes was not considered in this study. Shorter times eases the processing time and memory use of the algorithm while allowing to parallelize it in smaller processing threads. Longer windows might, however, improve the performance results further.

We enforced the algorithm to decompose the signal into two sources: one corresponding to SN, and the other corresponding to the heart pulse wave. The spectral contribution for SN events often showed a peak between 30 to $80 \mathrm{~Hz}$. We also found spectral components below but always accompanied with higher frequency components similar to Fig. 4. In our previous study [32], we found statistical differences in the bands $6-16 \mathrm{~Hz}$ and $60-100 \mathrm{~Hz}$. These spectral components in $6-16 \mathrm{~Hz}$ are very likely to be related to increased efforts, which often appear concurrently with SN. Norman et al. [36] used the spectral band $20-30 \mathrm{~Hz}$ to evaluate SN events. However, in our experiments, several SN profiles did not contain spectral components only in this band. Another study performed with PVDF sensors by Hwang et al. used a power ratio between components above and below $10 \mathrm{~Hz}$ [44]. Judging from the results shown in Fig. 4, we can verify that some snore events have spectral components lower than and above $30 \mathrm{~Hz}$ (e.g. Fig. 3b). These spectral components can be an indicator of the SN type and points a direction of the applicability of this method. In particular, the ability of the algorithm to show the spectral profile of the snores, could categorize SN based on the vibration spectral profile, e.g. oral and nasal. This method is more powerful than obtaining a mean PSD for each SN epoch, i.e. isolating events by source separation, yields a better representation of SN when compared with different feature sets (e.g. relative power or power ratios), which does not adapt as well to the various types of SN events.

Snoring detection using other sensors have been performed with higher sensitivity (usually above 90\%) [64]-[67] in very heterogeneous cohorts and by using diverse signal processing methods. The Emfit mattress offers advantages over other sensors. In situations where snoring is oral, nasal prongs are not useful to capture SN. Also, moisture in the nasal prongs produces high frequency components that might be interpreted as SN. Furthermore, piezoelectric sensors have the known issue that may entirely or partially detach during the night due to movement and sweat, leaving this sensor useless for SN detection. In all these cases, alternative signals are needed. On the other hand, mattress sensor like Emfit will detect the SN regardless the SN type, i.e. oral or nasal by using the obtained spectral profiles. Furthermore, despite the fact that external microphones offer a high-quality measure of the characteristics of SN, its setting is complex and dependent on the microphone, distance from the patient, and relative position of the patients with the microphone. The Emfit mattress does not detect as high frequency components like microphones; but it does detect low frequency vibrations produced by the partial obstruction of the airways, which may have a physiological impact on the surrounding tissues [15][18].

Snoring detection using bed mattresses has been approached in by Hwang et al. [44]. Compared to their method, the presented approach adapts to the SN pattern of each subject and isolates SN from the main signal. Further, this method does not require training.

The sensitivity to noise and artifacts is one of the main weaknesses of the method. Proper segmentation and artifact detection could be used to further improve the results; artifacts may include, breathing events, movements, coughing, and breathing efforts. On the other hand, we modeled the presence of events or snores with a generalized extreme value and Gaussian mixture to further analyze the epoch versus a gamma distribution. Other approaches could yield better results to classify the epoch; for example, convolutional neural networks. However, they require training which was out of the scope of this contribution.

Our algorithm showed diverse results when applied to the dataset. Its performance in some of the patients was notably worse. After inspecting these patients, the worse performances were due to the low signal intensity and high heterogeneous noise in the recorded Emfit signal. As shown by the RBCG and PSNR, the noise was more predominant on these signals. In particular, deeper inspection on subject Id. 18, showed high noise. Our tests proved that wavelet denoising improved results. Further research on preprocessing could be conducted to study the nature of the noise, and best approaches to remove it without affecting the time signal domain.

We also observed that some annotations were difficult to assess, i.e. it was not so clear whether the subject was snoring. Some events had clear high-frequency components in the nasal pressure signal. However, in the microphone nothing was heard. This is presumably due to humidity in the nasal 
prongs. Other SN events were sometimes considered SN where in fact was strong breathing. The Emfit did, however, detect these situations where vibrations occurred.

Since BCG must be present in all recordings we assumed the median spectral BCG magnitude in the $6-10 \mathrm{~Hz}$ band as a reference. Results could be improved by using $\mathrm{R}_{\mathrm{BCG}}$ to estimate the threshold $\left(\lambda_{t h}\right)$. Also, instead of normalizing the spectrogram, the intensity of the vibrations in conjunction with $\mathrm{R}_{\mathrm{BCG}}$ could be used as an objective measurement of $\mathrm{SN}$, which could allow comparing inter-subject SN intensity.

\section{CONCLUSIONS}

In this paper, we presented a method which can be used for snoring event detection, the studying of the spectral profile of

\section{REFERENCES}

[1] C. Guilleminault and A. Bassiri, "Clinical Features and Evaluation of Obstructive Sleep Apnea-Hypopnea Syndrome and Upper Airway Resistance Syndrome,” in Principles and Practice of Sleep Medicine, Elsevier, 2005, pp. 1043-1052.

[2] D. J. Gottlieb, Q. Yao, S. Redline, T. Ali, and M. W. Mahowald, "Does snoring predict sleepiness independently of apnea and hypopnea frequency?,” Am J Respir Crit Care Med, vol. 162, no. 4, pp. 1512-1517, Oct. 2000.

[3] K. F. Whyte, M. B. Allen, A. A. Jeffrey, G. A. Gould, and N. J. Douglas, "Clinical features of the sleep apnoea/hypopnoea syndrome.," The Quarterly journal of medicine, vol. 72, no. 267, pp. 659-666, Jul. 1989.

[4] E. Lindberg, A. Taube, C. Janson, T. Gislason, K. Svärdsudd, and G. Boman, "A 10-year follow-up of snoring in men.," Chest, vol. 114, no. 4, pp. 1048-1055, Oct. 1998.

[5] S. Spörndly-Nees, P. Åsenlöf, J. Theorell-Haglöw, M. Svensson, H. Igelström, and E. Lindberg, "Leisure-time physical activity predicts complaints of snoring in women: a prospective cohort study over 10 years.," Sleep Med., vol. 15, no. 4, pp. 415-421, Apr. 2014.

[6] M. Svensson, K. A. Franklin, J. Theorell-Haglöw, and E. Lindberg, "Daytime sleepiness relates to snoring independent of the apnea-hypopnea index in women from the general population.," Chest, vol. 134, no. 5, pp. 919-924, Nov. 2008.

[7] E. Lindberg, A. Taube, C. Janson, T. Gislason, K. Svärdsudd, and G. Boman, "A 10-Year Follow-up of Snoring in Men,” Chest, vol. 114, no. 4, pp. 1048-1055, Oct. 1998.

[8] W. Beninati, C. D. Harris, D. L. Herold, and J. W. Shepard Jr, "The Effect of Snoring and Obstructive Sleep Apnea on the Sleep Quality of Bed Partners," Mayo Clinic Proceedings, vol. 74, no. 10, pp. 955-958, Oct. 1999.

[9] M. L. Jackson, M. E. Howard, and M. Barnes, "Cognition and daytime functioning in sleep-related breathing disorders.,” Prog Brain Res, vol. 190, pp. 5368, 2011.

[10] J. Ulfberg, N. Carter, M. Talbäck, and C. Edling, "Excessive daytime sleepiness at work and subjective snoring events, and for the estimation of the intensity of snoring by analyzing the signal obtaining from the Emfit sensor. The method could detect $82.81 \%$ of the snoring events with the positive predictive value of $86.29 \%$. The algorithm can adapt to individual snore profiles and thus, it can be easily applied in clinical practice.

\section{ACKNOWLEDGMENTS}

This study was financially supported by Vilho, Yrjö ja Kalle Väisälän foundation, and by Competitive State Research Financing of the Expert Responsibility area of Tampere University Hospital (Grants 9P013, 9R007, 9S007).

work performance in the general population and among heavy snorers and patients with obstructive sleep apnea.," Chest, vol. 110, no. 3, pp. 659-663, Sep. 1996.

[11] T. Young, J. Blustein, L. Finn, and M. Palta, "Sleepdisordered breathing and motor vehicle accidents in a population-based sample of employed adults.," Sleep, vol. 20, no. 8, pp. 608-613, Aug. 1997.

[12] E. J. Kezirian and J. L. Chang, "Snoring without OSA and Health Consequences: The Jury Is Still Out," Sleep, 2013.

[13] J. M. Marin, S. J. Carrizo, E. Vicente, and A. G. N. Agusti, "Long-term cardiovascular outcomes in men with obstructive sleep apnoea-hypopnoea with or without treatment with continuous positive airway pressure: an observational study.," vol. 365, no. 9464, pp. 1046-1053, Mar. 2005.

[14] J. Rich, A. Raviv, N. Raviv, and S. E. Brietzke, "An epidemiologic study of snoring and all-cause mortality.," Otolaryngol Head Neck Surg, vol. 145, no. 2, pp. 341346, Aug. 2011.

[15] D. Friberg, T. Ansved, K. Borg, B. Carlsson-Nordlander, H. Larsson, and E. Svanborg, "Histological indications of a progressive snorers disease in an upper airway muscle.,” Am J Respir Crit Care Med, vol. 157, no. 2, pp. 586-593, Feb. 1998.

[16] J.-G. Cho, P. K. Witting, M. Verma, B. J. Wu, A. Shanu, K. Kairaitis, T. C. Amis, and J. R. Wheatley, "Tissue vibration induces carotid artery endothelial dysfunction: a mechanism linking snoring and carotid atherosclerosis?,” Sleep, vol. 34, no. 6, pp. 751-757, Jun. 2011.

[17] F. Puig, F. Rico, I. Almendros, J. M. Montserrat, D. Navajas, and R. Farre, "Vibration enhances interleukin-8 release in a cell model of snoring-induced airway inflammation.," Sleep, vol. 28, no. 10, pp. 1312-1316, Oct. 2005.

[18] S. R. Govindaraju, J. L. Bain, T. J. Eddinger, and D. A. Riley, "Vibration Causes Acute Vascular Injury in a Two- Step Process: Vasoconstriction and Vacuole Disruption,” The Anatomical Record, vol. 291, no. 8, pp. 999-1006, Aug. 2008.

[19] N. Chirakalwasan, D. L. Ruzicka, J. W. Burns, and R. D. Chervin, "Do snoring sounds arouse the snorer?," Sleep, vol. 36, no. 4, pp. 565-571, Apr. 2013.

[20] R. B. Berry, R. Brooks, and C. E. Gamaldo, "AASM 
Manual for the Scoring of Sleep and Associated Events; Rules, Terminology and Technical Specifications," vol. 8, no. 5. pp. 597-619, 15-Oct-2012.

[21] E. S. Arnardottir, B. Isleifsson, J. S. Agustsson, G. A. Sigurdsson, M. O. Sigurgunnarsdottir, G. T. Sigurđarson, G. Saevarsson, A. T. Sveinbjarnarson, S. Hoskuldsson, and T. Gislason, "How to measure snoring? A comparison of the microphone, cannula and piezoelectric sensor,” J Sleep Res, vol. 25, no. 2, pp. 1-11, Nov. 2015.

[22] M. Paajanen, H. Välimäki, and J. Lekkala, "Modelling the electromechanical film (EMFi)," Journal of Electrostatics, vol. 48, no. 3, pp. 193-204, Mar. 2000.

[23] S. Rajala and J. Lekkala, "Film-Type Sensor Materials PVDF and EMFi in Measurement of Cardiorespiratory Signals-A Review," Sensors Journal, IEEE, vol. 12, no. 3, pp. 439-446, Mar. 2012.

[24] C. Brüser, K. Stadlthanner, S. de Waele, and S. Leonhardt, "Adaptive beat-to-beat heart rate estimation in ballistocardiograms.," IEEE Trans Inf Technol Biomed, vol. 15, no. 5, pp. 778-786, Aug. 2011.

[25] C. Brüser, S. Winter, and S. Leonhardt, "Robust interbeat interval estimation in cardiac vibration signals.," Physiol. Meas., vol. 34, no. 2, pp. 123-138, Jan. 2013.

[26] C. Brüser, J. M. Kortelainen, S. Winter, M. Tenhunen, J. Pärkkä, and S. Leonhardt, "Improvement of ForceSensor-Based Heart Rate Estimation Using Multichannel Data Fusion,” IEEE J Biomed Health Inform, vol. 19, no. 1, pp. 227-235, 2014.

[27] J. Paalasmaa and M. Ranta, "Detecting heartbeats in the ballistocardiogram with clustering," presented at the Proc. ICML/UAI/COLT Workshop Mach. Learn. HealthCare Appl., Helsinki, Finland, 2008, 2008, vol. 9.

[28] J. Paalasmaa, H. Toivonen, and M. Partinen, “Adaptive Heartbeat Modelling for Beat-to-beat Heart Rate Measurement in Ballistocardiograms.," IEEE J Biomed Health Inform, vol. 19, no. 6, pp. 1945-1952, Mar. 2014.

[29] Y. Chee, J. Han, J. Youn, and K. Park, "Air mattress sensor system with balancing tube for unconstrained measurement of respiration and heart beat movements.," Physiol. Meas., vol. 26, no. 4, pp. 413-422, Aug. 2005.

[30] M. Tenhunen, E. Rauhala, J. Virkkala, O. Polo, A. Saastamoinen, and S.-L. Himanen, "Increased respiratory effort during sleep is non-invasively detected with movement sensor.," Sleep Breath, vol. 15, no. 4, pp. 737-746, Nov. 2011.

[31] M. Tenhunen, E. Elomaa, H. Sistonen, E. Rauhala, and S.-L. Himanen, "Emfit movement sensor in evaluating nocturnal breathing.," Respiratory Physiology \& Neurobiology, vol. 187, no. 2, pp. 183-189, Jun. 2013.

[32] J. M. Perez-Macias, J. Viik, A. Värri, S.-L. Himanen, and M. Tenhunen, "Spectral analysis of snoring events from an Emfit mattress,” Physiol. Meas., vol. 37, no. 12, pp. 2130-2143, Nov. 2016.

[33] J. Alametsä, E. Rauhala, E. Huupponen, A. Saastamoinen, A. Värri, A. Joutsen, J. Hasan, and S.-L. Himanen, "Automatic detection of spiking events in EMFi sheet during sleep," Medical Engineering \& Physics, vol. 28, no. 3, pp. 9-9, Mar. 2006.

[34] O. Polo, "Partial upper airway obstruction during sleep. Studies with the static charge-sensitive bed (SCSB).," vol. 606, pp. 1-118, 1992.

[35] O. Polo, L. Brissaud, B. Sales, A. Besset, and M. Billiard, "The validity of the static charge sensitive bed in detecting obstructive sleep apnoeas.," Eur Respir J, vol. 1, no. 4, pp. 330-336, Mar. 1988.

[36] M. B. Norman, S. Middleton, O. Erskine, P. G. Middleton, J. R. Wheatley, and C. E. Sullivan, "Validation of the Sonomat: a contactless monitoring system used for the diagnosis of sleep disordered breathing.," Sleep, vol. 37, no. 9, pp. 1477-1487, Sep. 2014.

[37] S. H. Hwang, H. J. Lee, H. N. Yoon, D. W. Jung, Y.-J. G. Lee, Y. J. Lee, D.-U. Jeong, and K. S. Park, "Unconstrained sleep apnea monitoring using polyvinylidene fluoride film-based sensor.,” IEEE Trans. Biomed. Eng., vol. 61, no. 7, pp. 2125-2134, Jul. 2014.

[38] T. Agatsuma, K. Fujimoto, Y. Komatsu, K. Urushihata, T. Honda, T. Tsukahara, and T. Nomiyama, "A novel device (SD-101) with high accuracy for screening sleep apnoea-hypopnoea syndrome,” Respirology, vol. 14, no. 8, pp. 1143-1150, Nov. 2009.

[39] M. Arimoto, T. Shiomi, R. Sasanabe, S. Inagawa, H. Ueda, and S. Inafuku, "A sheet- type device for homemonitoring sleep apneas in children," Sleep and Biological Rhythms, vol. 9, no. 2, pp. 103-111, Apr. 2011.

[40] J. H. Shin, Y. J. Chee, D.-U. Jeong, and K. S. Park, "Nonconstrained sleep monitoring system and algorithms using air-mattress with balancing tube method.," TITB, vol. 14, no. 1, pp. 147-156, Jan. 2010.

[41] A. P. Narechania, I. I. Garić, I. Sen-Gupta, M. P. Macken, E. E. Gerard, and S. U. Schuele, "Assessment of a quasi-piezoelectric mattress monitor as a detection system for generalized convulsions.," Epilepsy Behav, vol. 28, no. 2, pp. 172-176, Aug. 2013.

[42] K. V. Poppel, S. P. Fulton, A. McGregor, M. Ellis, A. Patters, and J. Wheless, "Prospective Study of the Emfit Movement Monitor," Journal of Child Neurology, vol. 28, no. 11, pp. 1434-1436, Oct. 2013.

[43] E. Rauhala, J. Virkkala, and S.-L. Himanen, "Periodic limb movement screening as an additional feature of Emfit sensor in sleep-disordered breathing studies," J Neurosci Methods, vol. 178, no. 1, pp. 157-161, Dec. 2008.

[44] S. H. Hwang, C. M. Han, H. N. Yoon, D. W. Jung, Y. J. Lee, D.-U. Jeong, and K. S. Park, "Polyvinylidene fluoride sensor-based method for unconstrained snoring detection.," Physiol. Meas., vol. 36, no. 7, pp. 13991414, Jul. 2015.

[45] J. C. B. Paris Smaragdis, "Non-Negative Matrix Factorization for Polyphonic Music Transcription,” pp. 1-4, Sep. 2003.

[46] T. Virtanen, "Monaural Sound Source Separation by Nonnegative Matrix Factorization With Temporal Continuity and Sparseness Criteria,” IEEE Transactions on Audio, Speech and Language Processing, vol. 15, no. 3, pp. 1066-1074, 2007.

[47] G. Shah, P. Koch, and C. B. Papadias, "On the blind recovery of cardiac and respiratory sounds.," IEEE J Biomed Health Inform, vol. 19, no. 1, pp. 151-157, Dec. 
2014.

[48] M. R. Keshtkaran and Z. Yang, “A Fast, robust algorithm for power line interference cancellation in neural recording," arXiv.org, vol. cs.SY, no. 2. p. 026017, 27Feb-2014.

[49] S. R. Messer, J. Agzarian, and D. Abbott, "Optimal wavelet denoising for phonocardiograms," Microelectronics Journal, vol. 32, no. 12, pp. 931-941, Dec. 2001.

[50] N. Nikolaev, Z. Nikolov, A. Gotchev, and K. Egiazarian, "Wavelet domain Wiener filtering for ECG denoising using improved signal estimate," presented at the 2000 International Conference on Acoustics, Speech and Signal Processing, vol. 6, pp. 3578-3581.

[51] F. R. Hampel, "The Influence Curve and its Role in Robust Estimation,” Journal of the American Statistical Association, vol. 69, no. 346, pp. 383-393, Jun. 1974.

[52] M. F and T. JW, "Data analysis and regression: a second course in statistics.," Addison-Wesley Series in Behavioral Science: Quantitative Methods, 1977.

[53] L. Ottaviani and D. Rocchesso, "Separation of speech signal from complex auditory scenes," Proc COST G-6 Conf on Digital Audio Effects, 2001.

[54] P. Paatero and U. Tapper, "Positive matrix factorization: A non-negative factor model with optimal utilization of error estimates of data values," Environmetrics, vol. 5, no. 2, pp. 111-126, Jun. 1994.

[55] D. D. Lee and H. S. Seung, "Learning the parts of objects by non-negative matrix factorization," Nature, vol. 401, no. 6755, pp. 788-791, Oct. 1999.

[56] D. D. Lee and H. S. Seung, "Algorithms for Nonnegative Matrix Factorization,” pp. 556-562, 2001.

[57] T. Virtanen, J. F. Gemmeke, B. Raj, and P. Smaragdis, "Compositional Models for Audio Processing: Uncovering the structure of sound mixtures.," SPM, vol. 32, no. 2, pp. 125-144, 2015.

[58] P. Smaragdis, "Non-negative Matrix Factor Deconvolution; Extraction of Multiple Sound Sources from Monophonic Inputs.," ICA, vol. 3195, no. 63, pp. 494-499, 2004.

[59] A. Savitzky and M. J. E. Golay, "Smoothing and Differentiation of Data by Simplified Least Squares Procedures.," Analytical Chemistry, vol. 36, no. 8, pp. 1627-1639, Jul. 1964.

[60] C. M. Hurvich and C. L. Tsai, "Regression and time series model selection in small samples," Biometrika, vol. 76, no. 2, pp. 297-307, 1989.

[61] K. P. Burnham and D. R. Anderson, Model Selection and Inference. New York, NY: Springer Science \& Business Media, 2013.

[62] J. M. A. Tanskanen, F. E. Kapucu, and J. A. K. Hyttinen, "On the threshold based neuronal spike detection, and an objective criterion for setting the threshold," presented at the 2015 7th International IEEE/EMBS Conference on Neural Engineering (NER, 2015, pp. 1016-1019.

[63] G. Grindlay, NMFLib-Efficient Matlab library implementing a number of common NMF variants. URLhttp://www. ee. columbia. edu/grindlay/code. html, 2010.

[64] M. Cavusoglu, M. Kamasak, O. Erogul, T. Ciloglu, Y. Serinagaoglu, and T. Akcam, "An efficient method for snore/nonsnore classification of sleep sounds," Physiol. Meas., vol. 28, no. 8, pp. 841-853, 2007.

[65] H.-K. Lee, J. Lee, H. Kim, J.-Y. Ha, and K.-J. Lee, "Snoring detection using a piezo snoring sensor based on hidden Markov models.,” Physiol. Meas., vol. 34, no. 5, pp. N41-9, May 2013.

[66] A. Yadollahi and Z. Moussavi, "Automatic breath and snore sounds classification from tracheal and ambient sounds recordings,” Medical Engineering \& Physics, vol. 32, no. 9, pp. 985-990, Nov. 2010.

[67] E. Dafna, A. Tarasiuk, and Y. Zigel, “Automatic detection of whole night snoring events using noncontact microphone.," PLoS One, vol. 8, no. 12, p. e84139, 2013. 